\title{
Molecular Interaction between Bacterial Antigens and Macrophage Receptors Studied by Atomic Force Microscopy
}

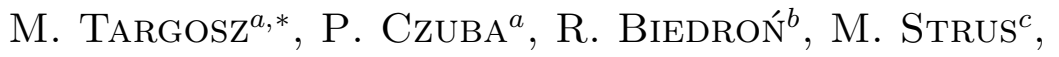 \\ A. GAmian ${ }^{d}$, J. MARCINKIEWICZ ${ }^{b}$ AND M. SzYMOŃSKI ${ }^{a}$ \\ ${ }^{a}$ Institute of Physics, Jagiellonian University \\ Reymonta 4, 30-059 Kraków, Poland \\ ${ }^{b}$ Institute of Immunology, Medical College, Jagiellonian University \\ Czysta 17, 31-121 Kraków, Poland \\ ${ }^{c}$ Institute of Microbiology, Medical College, Jagiellonian University \\ Czysta 17, 31-121 Kraków, Poland \\ ${ }^{d}$ Institute of Immunology and Experimental Therapy, Polish Academy of Sciences \\ Weigla 12, 53-114 Wrocław, Poland \\ Atomic force spectroscopy was used to study interaction strengths be- \\ tween bacterial antigens and receptors on macrophages. This method al- \\ lowed for a direct comparison of the interaction strengths in different systems \\ studied at the level of single molecules. \\ PACS numbers: 82.37.Rs, 87.64.Dz, 87.15.Kg
}

\section{Introduction}

Atomic force microscopy (AFM) is a high resolution imaging method for the investigation of biological samples. In particular it allows studies of dynamical biological processes in vivo [1]. In addition to its microscopic capabilities, AFM allows direct probing of the specific non-covalent forces between isolated biological molecules [2-5], as well as between molecules and receptors on the surface of cells (e.g. ligand-receptor interactions) [6, 7]. A spontaneous dissociation rate of the molecular complex is increased by the mechanical load applied by the cantilever. This was discussed for the first time by Bell [8] and by Evans and Ritchie in terms of the force spectroscopy $[9,10]$. The rupture of the ligand-receptor bond is a fully statistical process and it always gives a continuous distribution of the

*corresponding author; e-mail: mtargosz@if.uj.edu.pl 
rupture. For investigations of energetic landscapes of a particular complex, series of measurements have to be performed, each corresponding to different cantilever retracting speed. However, assuming that the characteristic interaction lengths do not change significantly for different complexes, one may calculate the ratio of the corresponding molecular affinities by a comparison of mean rupture forces of various complexes. In addition, the frequency of the specific bond occurrences (so-called adhesion probability) directly provides information about the amount of particular receptors on the investigated surface.

In this work interactions between bacterial antigens and receptors on the macrophage surfaces are studied by means of atomic force spectroscopy. Three types of bacterial antigens extracted from different groups of bacterial cells are used: lipopolysaccharides (LPS), peptidoglycan (PGN), and exopolysaccharides (EPS) $[11,12]$. These bacterial antigens are located on the surfaces of both $\operatorname{Gm}(-)$ and $\mathrm{Gm}(+)$ bacteria and are responsible for inflammation processes. The activation of macrophages is a general feature of early stages of infection. The macrophages can recognise bacteria, kill them by a phagocythosis, and then produce some mediate particles (cytokines) that subsequently activate the immunological system. The macrophage has specialised pattern-recognition receptors on its surface specific to bacterial antigens: e.g. TLR4, TLR2. It is known $[13,14]$ that TLR4 is responsible for the bio-recognition of the LPS by the macrophages. TLR2 has been identified as a receptor for PGN. It is not known exactly, which receptor on macrophage, TLR2 or TLR4, binds EPS. Thus, the strength of interaction between these antigens and receptors is crucial for the response of the immunological protection system.

\section{Materials and method}

Bacterial antigens were attached to the AFM tips with the use of a chemical linker: 3-aminopropylthiethoxysilane (APTES). We used standard V-shaped silicon nitride $\left(\mathrm{Si}_{3} \mathrm{~N}_{4}\right)$ cantilevers (Microlevers, Veeco) with a nominal spring constant of $0.01 \mathrm{~N} / \mathrm{m}$. The value of loading rate was of $20 \mathrm{nN} / \mathrm{s}$. The macrophage cells were take from peritoneum of mouse, then spin-dried two times and suspended with $2 \%$ Bovie serum albumine (BSA) and Dulbecco's phosphate buffered saline (dPBS) solution. Next, they were put on glass coverslip and incubated for $2 \mathrm{~h}$ at $37^{\circ} \mathrm{C}$ conditions. Afterwards, the glass coverslip with macrophage cells was washed in dPBS and immediately taken for measurements. Two types of macrophage cells were used: cells from CBA mouse with all active receptors, and cells with inactive TLR4 receptor extracted from genetically modified mouse C3H/J.

The rupture forces characteristic of a particular complex are determined from a so-called "force vs. distance" curves. Examples of the force-distance curves showing single and multiple bond breaking (jumps) are presented in Fig. 1. LPS, PGN, and EPS have polymer structures [11, 12], therefore we can try to distinguish between specific and non-specific curves. The specific curves arising 

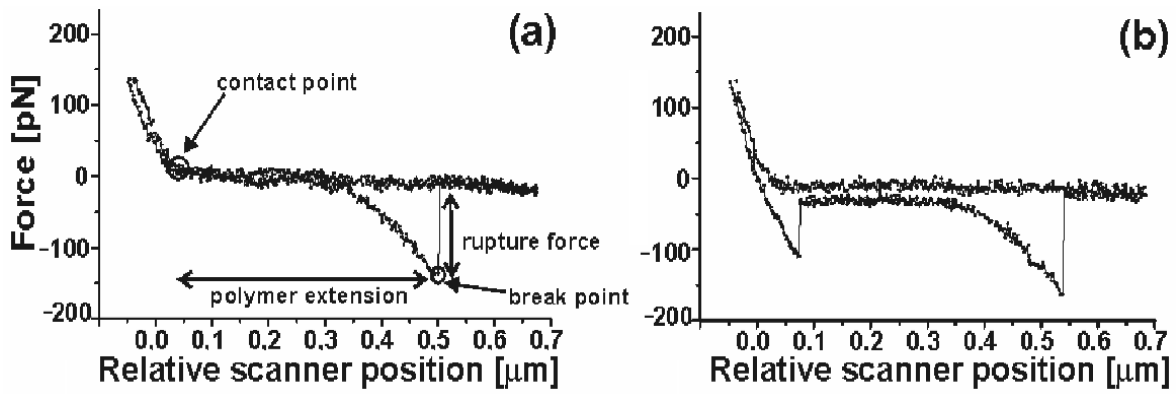

Fig. 1. Examples of force-distance curves measured for the bacterial antigen macrophage receptor systems. (a) The curve with a single adhesive jump and a characteristic polymer extension dependence indicating the occurrence of a specific bond. (b) The multiple bond curve. The first jump is assumed to come from a non-specific interaction, the second one with characteristic polymer extension shape is assumed to come from interaction between bacterial antigen and receptor on macrophage's cell. Let us note the different extraction of the transducer, which is used to differentiate between the specific and non-specific interactions [15].

from ligand-receptor interaction have a characteristic shape corresponding to the polymer extension. The distance between contact point and breaking point can be thus related to the length of polymer chain. On the basis of characteristic jumps (break points), visible in the force-distance curves (Fig. 1), the rupture force value can be determined.

\section{Results and discussion}

Histograms of rupture forces measured for the studied systems are presented in Fig. 2. For a complex: PGN covered tip-cell receptors with all active receptors (Fig. 2a), strong and frequent specific interactions can be observed. In this system we can suppose that the biggest contribution to interaction comes from the interaction of PGN with TLR2 receptor [13, 14]. The histogram is composed of three peaks. The first peak comes from weak specific interactions, i.e. interactions with receptors having a smaller affinity to PGN. This result shows that a weak TLR4-PGN interaction can take place. The second peak is attributed to a strong specific interaction PGN with TLR2 receptors (high affinity), whereas the third peak is attributed to double bond breaking. The histogram for system with the genetically modified cells (without TLR4 receptor) shows a single peak. This peak can be correlated with the second peak from the histogram of the previous system. In PGN-cell without active TLR4 receptors system the weak specific interaction, observed in the previous PGN-native cells system, was totally suppressed. We can conclude that those peaks are due to specific interactions between PGN-TLR2 receptors. Biochemical methods proved that PGM binds to TLR2 receptors, how- 

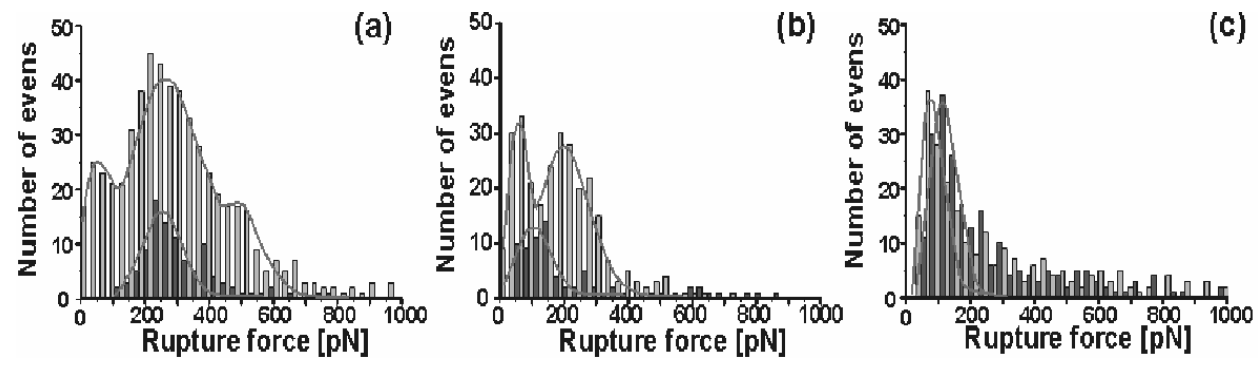

Fig. 2. Histograms of rupture forces obtained for antigen-macrophage receptor systems. (a) Histogram for PGN covered tip - native cell receptor complex is shown using bright bars and the histogram for PGN covered tip - genetically modified cells receptors (without TLR4) using dark bars. (b) Histogram for LPS covered tip - native cell receptor complex (bright bars) compared to the histogram for LPS covered tip - genetically modified cell receptors (dark bars). (c) EPS covered tip - native cell receptors (bright bars), EPS covered tip - genetically modified cell receptors (dark bars).

ever the competing interaction with TLR4 was not clear. Our results indicate the role of the TLR2 receptor in the PGN-macrophage bio-recognition.

Histograms obtained for: LPS-native cell complexes and LPS-genetically modified cell (without TLR4) complexes are compared in Fig. 2b. In the first system TLR4 is responsible for the bio-recognition of the LPS by the macrophages. For native cells the strong specific interaction is found. The histogram made for this case is composed of two peaks. Similarly to the previous system, the second peak is attributed to specific interactions with TLR4 receptor. These results are confirmed by the second histogram obtained for the LPS-cell with deactivated TLR4 receptors system. The histogram made for this system shows a single peak, coinciding with the first peak of the native system. As expected

TABLE

Adhesion probabilities $\left(P_{\mathrm{A}}\right)$ and rupture forces $\left(F_{\mathrm{R}}\right)$ determined from the histograms for studied complexes. The values of $F_{\mathrm{R}}$ are determined from Gaussian fits to the histograms. $A_{\%}$ - fractions of the subcomponents in the case of the CBA cell - PGN complex and CBA cell - LPS complex.

\begin{tabular}{|c|c|c|c|c|c|c|c|c|c|}
\hline & \multicolumn{4}{|c|}{ PGN } & \multicolumn{3}{|c|}{ LPS } & \multicolumn{2}{|c|}{ EPS } \\
\hline & \multicolumn{3}{|c|}{$F_{\mathrm{R}}[\mathrm{pN}] / A_{\%}$} & \multirow[t]{2}{*}{$P_{\mathrm{A}}$} & \multicolumn{2}{|c|}{$F_{\mathrm{R}}[\mathrm{pN}] / A_{\%}$} & \multirow[t]{2}{*}{$P_{\mathrm{A}}$} & \multirow[t]{2}{*}{$F_{\mathrm{R}}[\mathrm{pN}]$} & \multirow[t]{2}{*}{$P_{\mathrm{A}}$} \\
\hline & $\begin{array}{c}1 \text { st } \\
\text { peak }\end{array}$ & $\begin{array}{l}\text { 2nd } \\
\text { peak }\end{array}$ & $\begin{array}{c}\text { 3rd } \\
\text { peak }\end{array}$ & & $\begin{array}{c}1 \text { st } \\
\text { peak }\end{array}$ & $\begin{array}{c}\text { 2nd } \\
\text { peak }\end{array}$ & & & \\
\hline $\begin{array}{c}\text { cells from } \\
\text { CBA }\end{array}$ & $\begin{array}{c}67 \pm 8 \\
25 \%\end{array}$ & $\begin{array}{c}273 \pm 7 \\
60 \%\end{array}$ & $\begin{array}{c}503 \pm 15 \\
15 \%\end{array}$ & 0.6 & $\begin{array}{c}62 \pm 6 \\
25 \%\end{array}$ & $\begin{array}{c}212 \pm 5 \\
75 \%\end{array}$ & 0.55 & $100 \pm 4$ & 0.7 \\
\hline $\begin{array}{c}\text { cells from } \\
\text { C3H/J }\end{array}$ & - & $256 \pm 6$ & - & 0.3 & $104 \pm 4$ & - & 0.2 & $112 \pm 3$ & 0.7 \\
\hline
\end{tabular}


for TLR4 deactivated receptor cells, the specific interaction is almost completely faded out.

In Fig. 2c the rupture force histograms for EPS-native cell complexes and for EPS-genetically modified cell complexes are shown. Contrary to the results obtained for the previous systems, no differences in the histograms are observed. Till now it has been believed that EPS is a rival of LPS receptor on a macrophage cell. The histograms presented in Fig. 2c (through a direct comparison with the histogram of Fig. 2b) show that: (i) EPS-macrophage interaction is weaker than LPS-macrophage interaction (Fig. 2b); (ii) the EPS interaction with both type of macrophage cells (with and without TLR4) is comparable, therefore one can conclude that TLR4 does not (or does very weakly) contribute to the EPS bonding. These points indicate that there is no competition between both types of the antigens. Table summarizes the results of the above analysis.

\section{Conclusion}

Standard immunological methods provide information about ligand-receptor interactions by monitoring the products of these reactions on macroscopic scale. By using atomic force spectroscopy we are able to determine the strength of ligandreceptor interaction at the level of single molecules.

\section{Acknowledgments}

This work was supported by the grants: 1 P03B 09828 and 3 T11E 03326 , from the State Committee for Scientific Research (MNII) of Poland.

\section{References}

[1] T. Strunz, K. Oroszlan, R. Schafer, H.J. Guntherodt, Proc. Natl. Acad. Sci. 96, 11277 (1999).

[2] U.G. Lee, Science 266, 771 (1994).

[3] P. Bougrand, Rep. Prog. Phys. 62, 921 (1999).

[4] E.F. Florin, T.V. Moy, H.E. Gaub, Science 264, 415 (1994).

[5] P. Hinterdorfer, W. Baumgartner, H.J. Gruber, K. Schilcher, H. Schindler, Proc. Natl. Acad. Sci. 93, 3477 (1996).

[6] U. Dammer, O. Popescu, P. Wagner, D. Anselmetti, H.J. Guntherodt, G.N. Misevic, Science 267, 1173 (1995).

[7] M. Lekka, P. Laider, J. Dulinska, M. Łabȩdź, G. Pyka, Eur. Biophys. J. 33, 644 (2004).

[8] G.I. Bell, Science 200, 618 (1978).

[9] E. Evans, K. Ritchie, Biophys. J. 72, 1541 (1997).

[10] E. Evans, A. Leung, W. Heinrich, C. Zhu, Proc. Natl. Acad. Sci. 101, 11281 (2004).

[11] T. Lipinski, Ch. Jones, X. Lemerciner, A. Korzeniowska-Kowal, M. Strus, J. Rybka, A. Gamian, P.B. Heczko, Carbohydrate Research 338, 605 (2003). 
[12] G. Mielnik, A. Gamian, W. Doroszkiewicz, FEMS Immunology and Medical Microbiology 31, 169 (2001).

[13] M.M. Monik, G.E. Hunninghake, Eur. Respir. J. 20, 210 (2002).

[14] M. Muroi, K. Tanamoto, Infection and Immunity 70, 6043 (2002).

[15] T.V. Ratto, K.C. Langry, R.E. Rudol, R.L. Balhorn, M.J. Allen, M.W. McElfresh, Biophys. J. 86, 2430 (2004). 